\title{
Measurement of instantaneous 2-D velocity field and local chemiluminescence in a premixed-spray flame by PIV and MICRO system
}

\author{
Shohji TSUSHIMA, Masaaki NEGORO, Hiroyasu SAITOH \\ Manabu FUCHIHATA, Fumiteru AKAMATSU and Masashi KATSUKI \\ Department of Mechanical Engineering, Osaka University \\ 2-1 Yamada-oka, Suita, Osaka 565-0871, JAPAN
}

\begin{abstract}
In this article, we demonstrate combined measurements of particle image velocimetry (PIV) and Multi-colour Integrated Cassegrain Receiving Optics (MICRO) in an attempt to observe characteristics of propagating flame in a premixed-spray stream. High-speed images recorded with an intensified CCD camera and cross-correlation PIV method showed the capability in obtaining instantaneous velocity fields in sooty spray flames, where liquid fuel of kerosene was supplied in the form of premixed spray. It enabled us to discuss the influence of fluid turbulent motion on the process of preferential flame propagation. Local chemiluminescence in flames detected by MICRO system was conditionally processed in terms of the distance from the spray boundary that was determined from visualized spray images. The time-averaged onedimensional structure obtained statistically in the direction of flame propagation showed that two distinct reaction peaks appeared on both sides of the spray boundary, which corresponded to the main vaporization region of a spray.
\end{abstract}

\section{INTRODUCTION}

Flame propagation in a spray stream is a unique character of spray combustion (Myers and Lefebvre, 1986; Roth et al., 1996). Intensive efforts have been made to investigate premixedlike behaviour of spray flames by numerous researchers (Hayashi et al., 1976; Richards and Lefebvre, 1989; Greenberg et al., 1996). Since spray flames are heterogeneous turbulent reacting two-phase flows, they include inherently complicated transient structures. Besides, inhomogeneity of spray characteristics in space and time yields further complexity in flame structure. To clarify the spray flame structure, therefore, instrumentation with high resolution in both space and time is strongly required.

In our recent experiments, we observed time-series behaviour of spray flames on a premixedspray burner using laser tomography consisting of a high-speed CCD camera and an Argon-ion laser sheet. We found that a portion of premixed-spray stream sometimes disappeared rapidly 


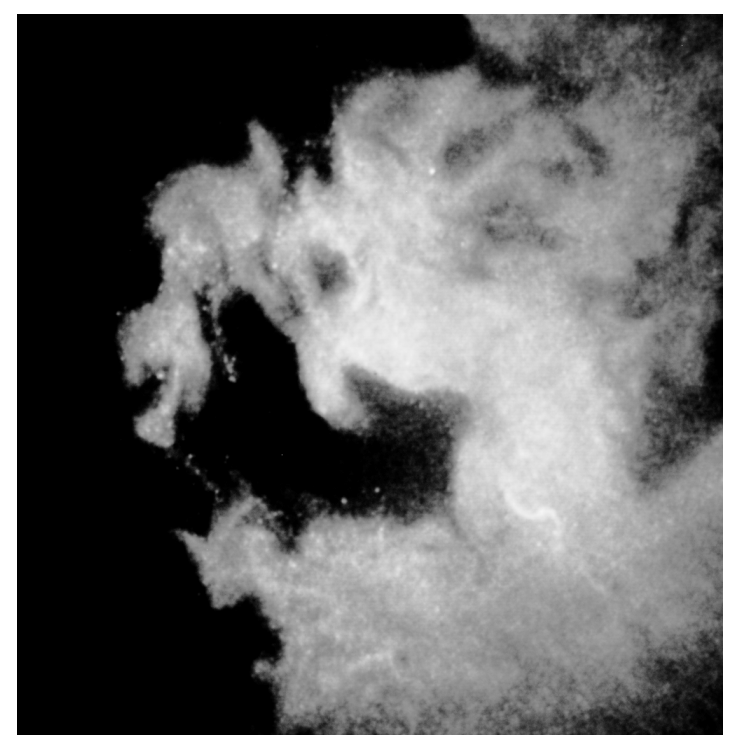

(a) Combusting

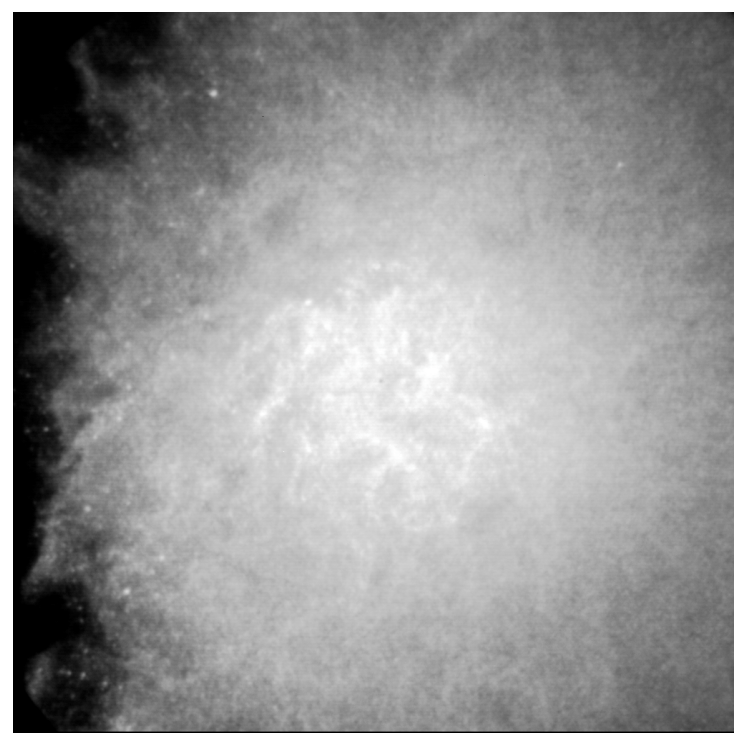

(b) Non-combusting

Figure 1 Typical cross-sectional image of spray stream.

in horizontal direction shown in Figure 1(a) (Tsushima et al., 1998). We thought that such a rapid disappearance of spray was caused by the fast propagation of flame preferentially intruding into the spray stream through the easy-to-burn region of the premixed-spray stream. However, since large scale turbulent motions in a shear layer may deform flame surfaces conspicuously as often seen in gaseous premixed turbulent flames (Chew et al., 1989), we could not deny that the rapid disappearance of a part of spray was caused by the large scale turbulent motion. Therefore, one of the objectives of the present study is to clarify whether a rapid disappearance of a part of spray stream was caused by the enhanced flame propagation or by the rapid displacement of droplets moving out of the probing laser sheet due to the turbulent motion.

In the present study, a cross-correlation PIV system consisting of an Argon-ion laser and a CCD camera coupled with an image intensifier was applied to the same premixed spray burner in order to obtain two-dimensional (2D) instantaneous velocity fields to clarify the influence of turbulent motion on flame propagation processes. To ensure the ability of the PIV system when it is applied to spray flames with bright luminosity, the obtained velocity fields were also compared with those measured by phase-Doppler analyzer (PDA).

Point-wise measurements of local chemiluminescence of $\mathrm{OH}$ and $\mathrm{CH}$ radicals together with Mie scattering from laser-illuminated droplets in the flame were also conducted simultaneously using MICRO system (Akamatsu et al., 1999) to examine the interlocation between propagating flames and spray boundaries. Further, the time-averaged one-dimensional flame structure in the direction of flame propagation was obtained statistically by combined data processing of both images and point-wise signals.

\section{EXPERIMENTAL APPARATUS}




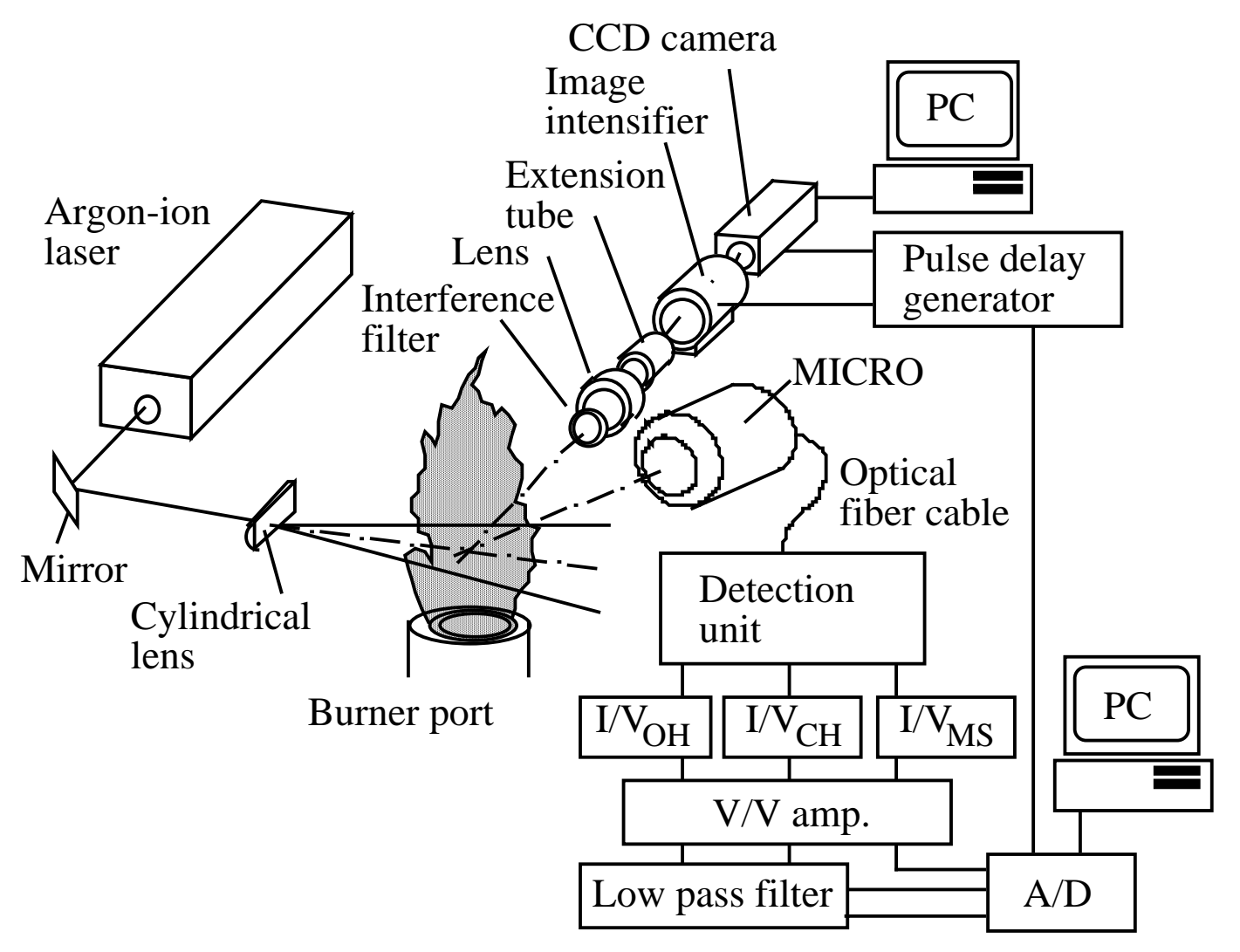

Figure 2 Experimental apparatus.

The experimental apparatus used in the study is shown in Figure 2. The details of premixedspray burner and flame photograph can be found elsewhere (Akamatsu et al., 1997). Flow conditions are thus briefly given here. Liquid fuel of kerosene was injected through an twinfluid atomizer which was placed $440 \mathrm{~mm}$ upstream from the burner port to form a premixedspray stream, almost uniformly suspended droplets in the air supplied as carrier gas. This unique configuration produces low slip velocity between suspended droplets and air because most of large droplets are eliminated in the mixing chamber (Akamatsu et al., 1997). The spray flame was anchored by a pilot burner of hydrogen diffusion flame formed around the burner port.

To illuminate fuel droplets, an Argon-ion laser (Spectra Physics, 2017) was used in the present study instead of a costly double-pulsed laser that is often used to freeze particle motion of interest in PIV measurements. A thin laser light sheet, $0.5 \mathrm{~mm}$ thickness, was formed by a cylindrical lens and illuminated a vertical plane above the burner port. Since we adopted continuous wave Argon-ion laser as a light source, an image intensifier with an electronic shutter (Hamamatsu, C6653) was utilized to control the exposure time. 
A CCD camera (Kodak, Megaplus Model ES 1.0) coupled with the image intensifier was used to record spray images. The "triggered dual exposure mode" originally installed in the camera takes two consecutive images with minimum separation time of $5 \mu \mathrm{s}$. The exposure time for the first image on the CCD camera is controllable up to $255 \mu \mathrm{s}$ by the "triggered dual exposure mode", and the exposure time for the second image is fixed at $33 \mathrm{~ms}$, which is too long for PIV imaging. Therefore, we have improved the sequence control for the electronic shutter of the camera to be used with a $\mathrm{CW}$ laser source. The pulse-timing diagram for PIV measurement is shown in Figure 3 together with the exposure time chart of the CCD camera. Totally three TTL signals generated by a pulse delay generator (PDG) (Stanford Research Systems, WC Model DG535) were used to obtain two consecutive images. The first TTL signal named "start-up pulse" is supplied to the CCD camera to trigger the acquisition of a pair of images. Image acquisition for the first frame is to start at least $20 \mu \mathrm{s}$ after the start-up pulse. Then, the second and the third pulse of TTL signals are supplied to the image intensifier to open the electronic shutter with the specified time duration, $\tau_{1}$ and $\tau_{2}$. The exposure time for the electronic shutter can be controlled by input pulse widths, $\Delta_{l}$ and $\Delta_{2}$. In the present study, the width and time duration of input TTL signals were carefully chosen in order to take two consecutive spray images with equal brightness. The adopted pulse sequence in Figure 3 shows the width of third pulse $2.7 \mu \mathrm{s}$, which is slightly shorter than that for the second pulse of

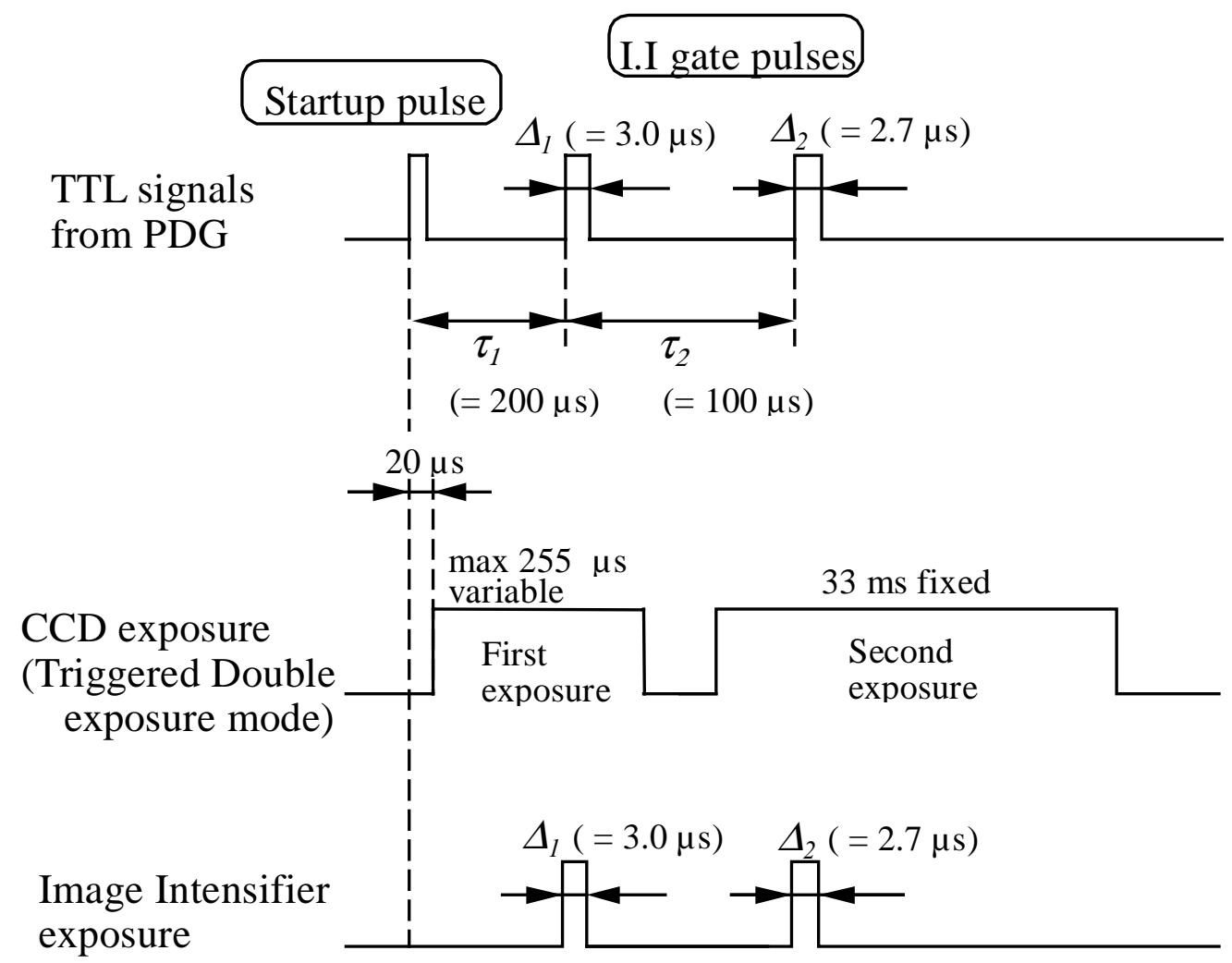

Figure 3 Pulse- and exposure-timing chart for PIV measurement using CW laser source. 
$3.0 \mu \mathrm{s}$. The delicate value was determined by try-and-error optimization to get two images of the same pixel brightness.

The area of image was a square of $30 \mathrm{~mm}$ by $30 \mathrm{~mm}$, that corresponds to $h=30 \mathrm{~mm}$ to $60 \mathrm{~mm}$ and $r=2 \mathrm{~mm}$ to $32 \mathrm{~mm}$, where $h$ and $r$ denote the axial and radial distance measured from the center of the burner port, respectively. Mie scattering signals from laser-illuminated droplets were collected onto 1008 pixels by 1018 pixels on CCD array through $514.5 \mathrm{~nm} \pm 1.8$ $\mathrm{nm}$ optical interference filter to eliminate spontaneous emissions from the flame. It should be noted that the displacement of droplets due to convection of the flow during the exposure time was negligible. The estimated displacement of droplets during two consecutive images from the measured axial mean velocity of droplets by a phase Doppler analyzer was approximately 20 pixels.

To derive instantaneous velocity fields of the premixed spray, the obtained image was firstly reduced into $1 / 3$-size frame to reduce calculation load on the computer. Consequently, the resultant spatial resolution of $1 / 3$ image was 336 pixels $\times 340$ pixels. Then, crosscorrelation method was applied to the reduced images. The size of reference circle for the calculation of cross-correlation used in the image processing was chosen at $1.5 \mathrm{~mm}$, which was equal to about 17 pixels.

Besides the PIV measurement, we carried out simultaneous measurement of Mie scattering from droplets as well as local chemiluminescence underlying in $\mathrm{OH}-$ and $\mathrm{CH}-\mathrm{band}$, the monitored wavelength of which was $308.5 \mathrm{~nm}$ and $430.5 \mathrm{~nm}$, respectively. Chemiluminescence was collected by the MICRO probe and guided into a detection unit through an optical fiber. Three sets of interference filter and photomultiplier (PMT) (Hamamatsu, $\mathrm{R} 106 \mathrm{UH})$ were allocated to select respective wavelength in the detection unit, and electric current signals from each PMT were amplified after conversion into voltage and digitized by an A/D converter (Elmec, EC-2390) with the sampling rate of $100 \mathrm{kHz}$. The more details of MICRO system can be found in literature (Akamatsu et al., 1999).

\section{RESULTS AND DISCUSSION}

\subsection{Influence of turbulence on preferential flame propagation}

One of the objectives of the present study is to clarify whether a rapid disappearance of a part of spray stream was caused by the enhanced flame propagation or by the rapid displacement of droplets moving out of the probing laser-sheet due to turbulent motions. In our previous observation on premixed-spray flames with a high-speed CCD camera (Tsushima et al., 1998), a part of spray boundary is frequently eroded into a concave-shaped boundary shown in Figure 1(a), referred as concavity hereafter. However, the formation of such concavity was not observed in non-combusting cases as seen in the Figure 1(b), in spite of the shear layer formed between the spray stream and the surrounding air. It was not clear enough from these pictures whether the rapid disappearance of a part of premixed-spray stream was caused by the rapid flame propagation or by turbulent fluid motions. 


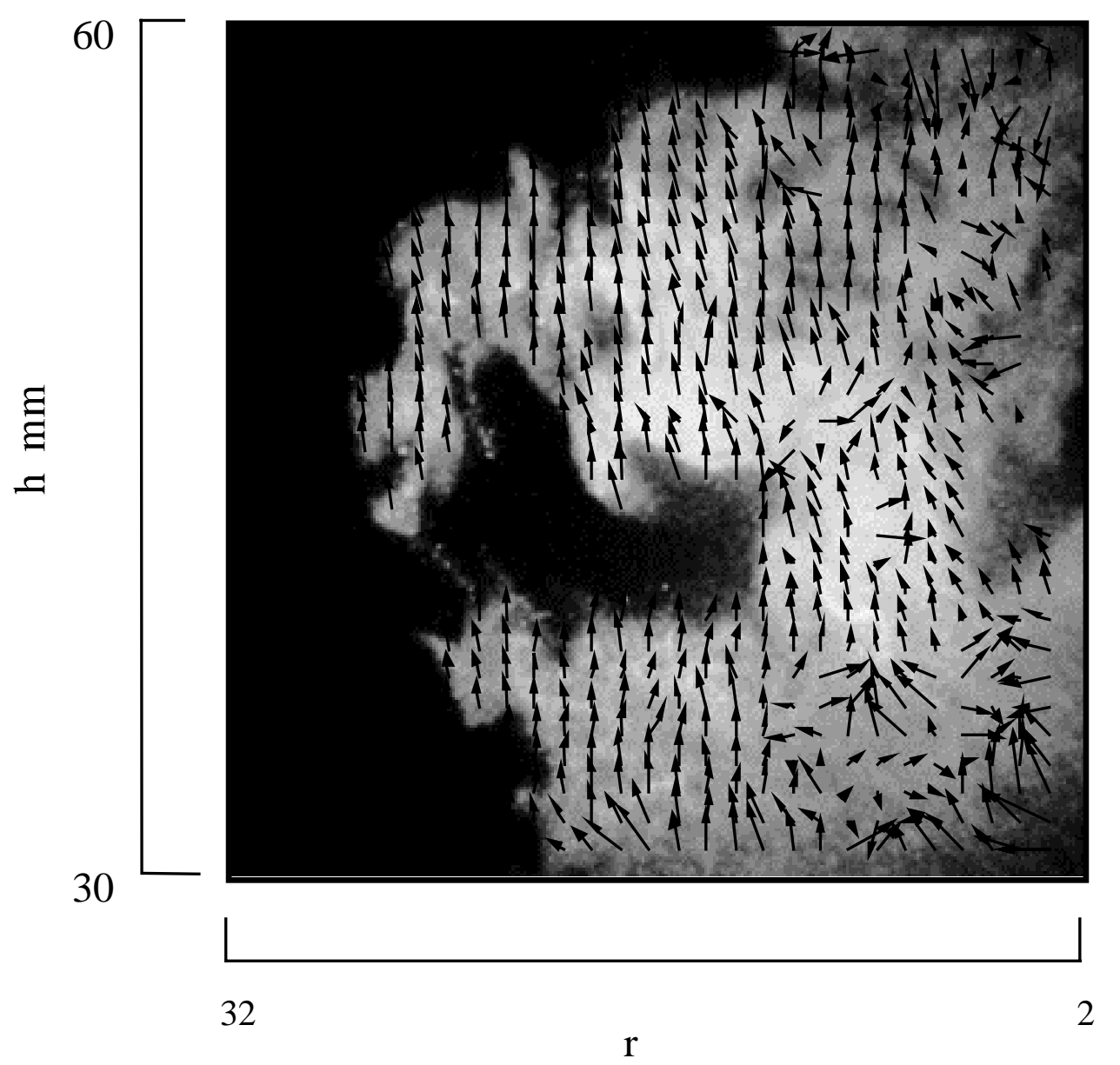

Figure 4 Visualized spray image and instantaneous velocity field inside the spray region.

Figure 4 exhibits a sample image of visualized spray in a premixed-spray flame where an instantaneous velocity field obtained by PIV is superimposed. The most of velocity vectors inside the spray region (white zone in the figure) have the same orientation as the bulk flow, that is, parallel to the $h$ axis. Adding to this fact, careful observation on velocity vectors particularly in the vicinity of periphery of the spray region have not identified any rotating motions associated with large eddies. Accordingly, turbulent motion does not seem to be an influencing factor on the rapid disappearance of a part of the premixed-spray stream. In order to verify the interpretation above, we should actually see that the rapid disappearance of a premixed-spray stream is caused by rapid vapourization of fuel droplets due to propagating flames, which will be explained in the later section.

As to verifying the measuring technique, velocity vectors obtained by PIV were compared with those measured by PDA system (Dantec, transmitter 60X; receiver 57X10 and signal processor 58N10). The number of sampled droplets at each measuring point in the PDA measurement was 3000, a few seconds was enough for the data acquisition at each point. In contrast, the presented results by PIV were obtained by averaging over 300 runs. Figure 5 


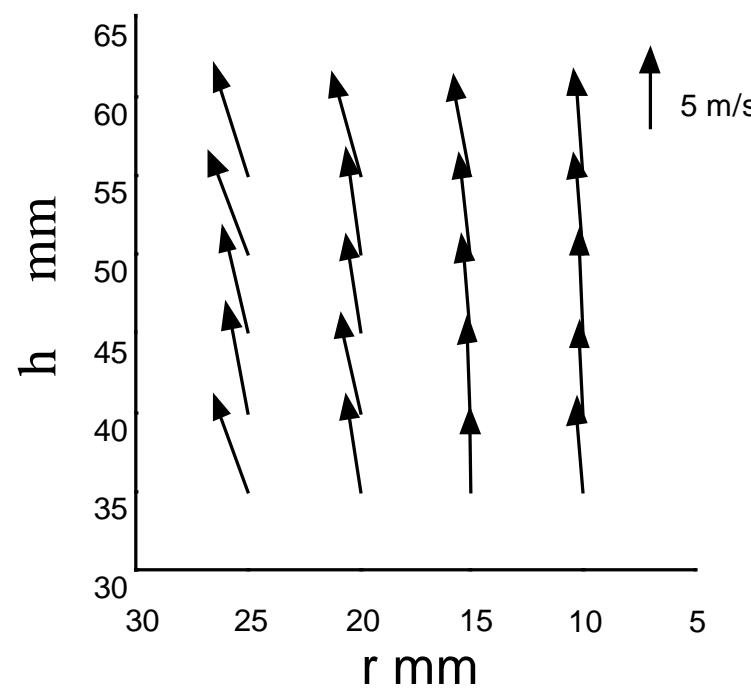

(a) PIV

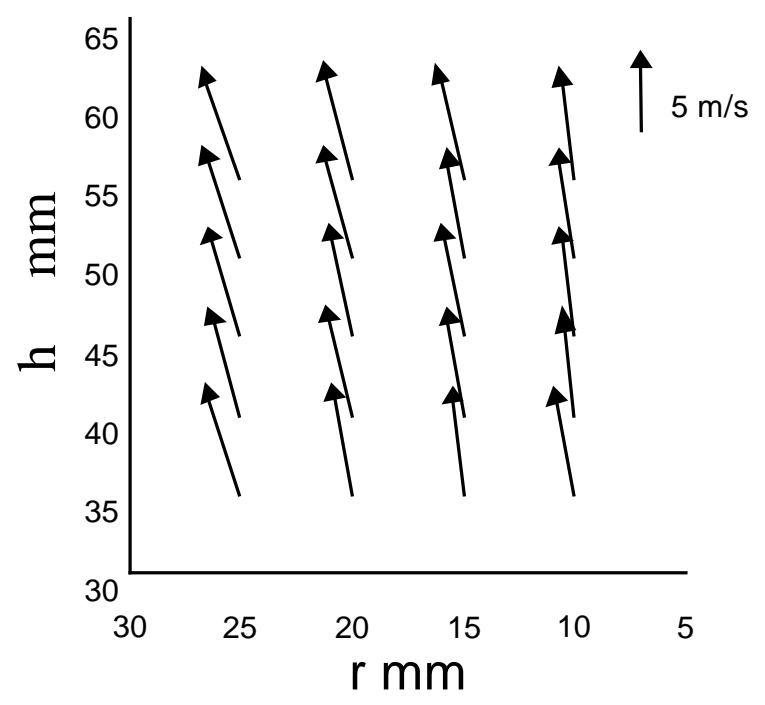

(b) PDA

Figure 5 Comparison of mean velocity vectors measured by PIV and PDA.

shows mean velocity vectors measured by PIV (Fig. 5 (a)) and PDA (Fig. 5(b)), respectively, which are in excellent agreement.

\subsection{Structure of propagating flame}

In this section, since we want to discuss the interlocation of reaction zone and spray boundary, we need to know the movement of spray boundary and the relative location of reaction zone with respect to the position of the instantaneous spray boundary. For the purpose, flame structure in the direction of propagation was examined by the combination of image and signal processing. First, we defined the instantaneous boundary of spray zone by binarizing each spray image. In these processes, the Gaussian intensity distribution of laser sheet was taken into account by changing the threshold of binarization for each horizontal pixel line taken from its probability density distribution of pixel intensity. From continuous signal of light emission detected at the focal point of MICRO placed in the plane of laser sheet, we can read out light emission intensities at the instant corresponding to the image. Figure 6 exemplifies the relation between the binarized spray boundary and the focal point of MICRO shown by a closed circle. Measured signal intensities of $\mathrm{OH}$ - and $\mathrm{CH}$-band emissions and Mie scattering at the instant of Figure 6 were $7.93 \mathrm{nA}, 308.3 \mathrm{nA}$ and $20.85 \mu \mathrm{A}$, respectively. As a result, reference distance from the focal point of MICRO probe to the spray boundary, $L$, can be determined $1.11 \mathrm{~mm}$ as illustrated in the figure.

The intensity distribution of each emission in terms of reference distance was obtained by averaging totally 300 runs in an attempt to deduce mean structure of spray flame in the direction of propagation. Figure 7 shows mean signal intensities of Mie scattering and $\mathrm{OH}$ and $\mathrm{CH}$-band emissions in terms of reference distance. The negative value of reference distance means that the measurement point of MICRO probe locates outside the spray boundary 


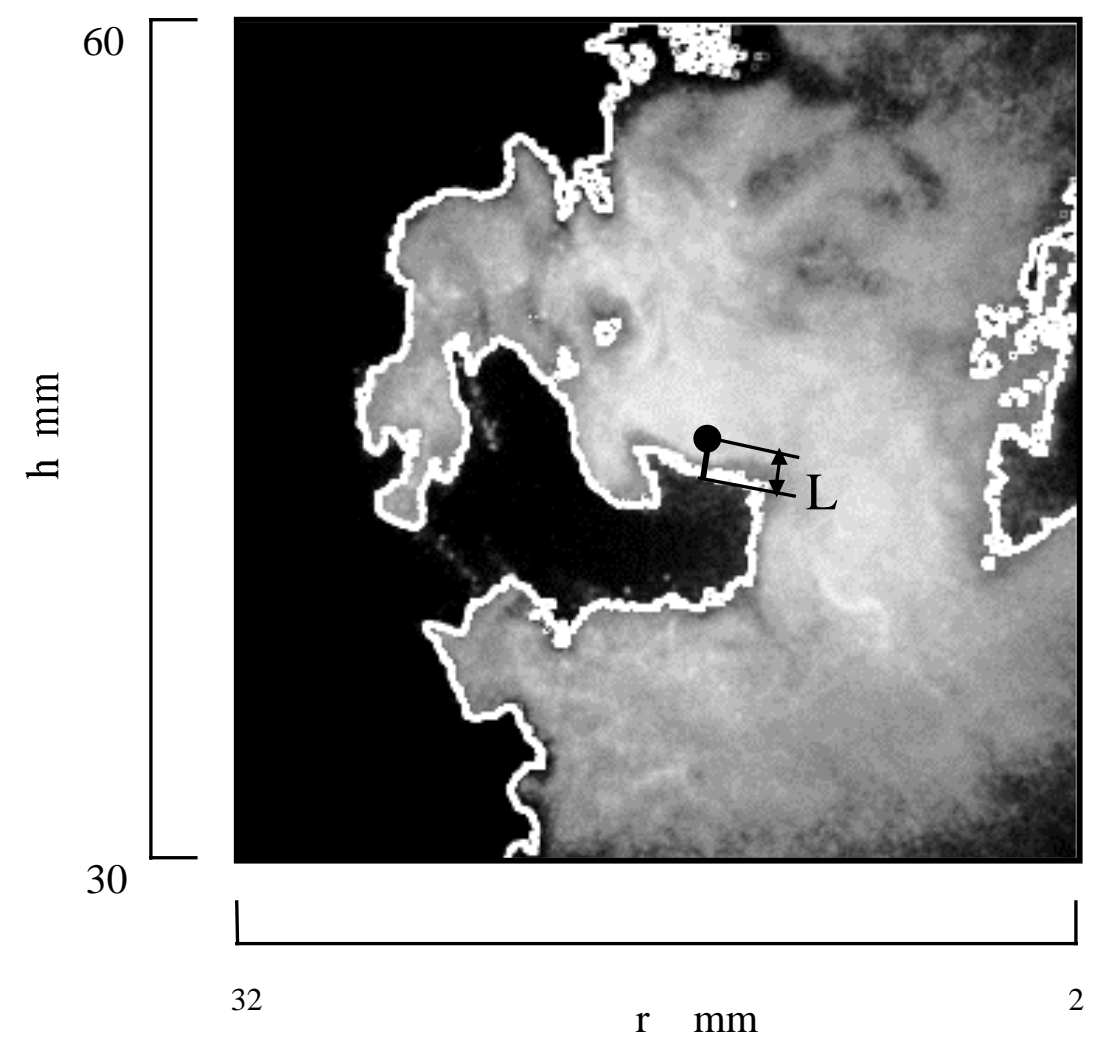

Figure 6 Binarized spray boundary and reference distance of the focal point of MICRO relative to the spray boundary.

where the number density of fuel droplet abruptly decreases due to vapourization. Judging from the figure, the intensity of Mie scattering shows the steepest decay at $L=0 \mathrm{~mm}$, and completely attenuates at $L=-2 \mathrm{~mm}$. This fact indicates that the evaporation of fuel droplets is at its maximum at the binarized spray boundary. Accordingly, the spray boundary defined by the image processing represents the main vaporization plane of premixed spray.

In Figure 7(b) and (c), two distinct peaks of $\mathrm{OH}$ - and $\mathrm{CH}$-band emissions can be identified. Physical interpretation on these chemiluminescence, which are considered to be good markers to identify the existence of reaction zone, are given in the literature (Gaydon, 1974). It shows that intensive reaction regions exist on both sides of the main vaporization plane. This sort of two-staged flame structure in spray flames was reported by Li and Williams (1996) and Continillo and Sirignano (1990). However, further investigations will be necessary to clarify the instantaneous structure of the propagating flame, since the demonstrated results in the present study are time-averaged.

\subsection{Movement of spray boundary}

Since the velocity measurement by PIV is based on the movement of particles suspended in the fluid, it loses the accuracy and physical background when it is applied to rapidly evaporating or disappearing particles. However, in order to prove the fact that the frequent disappearance of a 


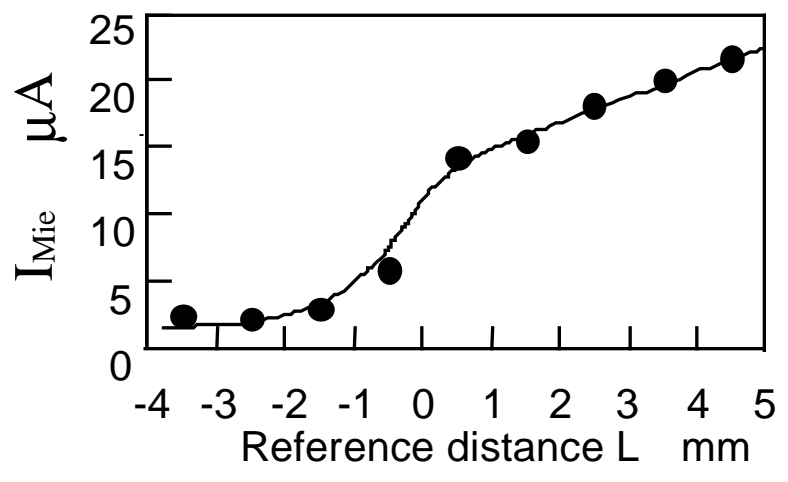

(a) Mie scattering

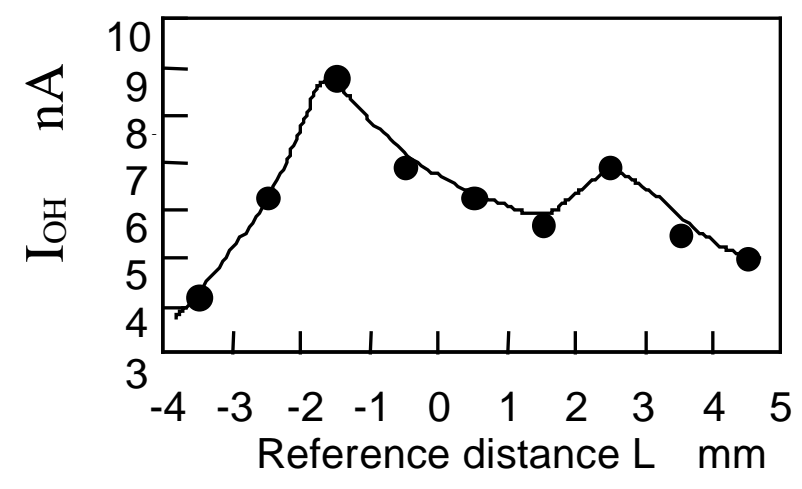

(b) $\mathrm{OH}$-band emissions

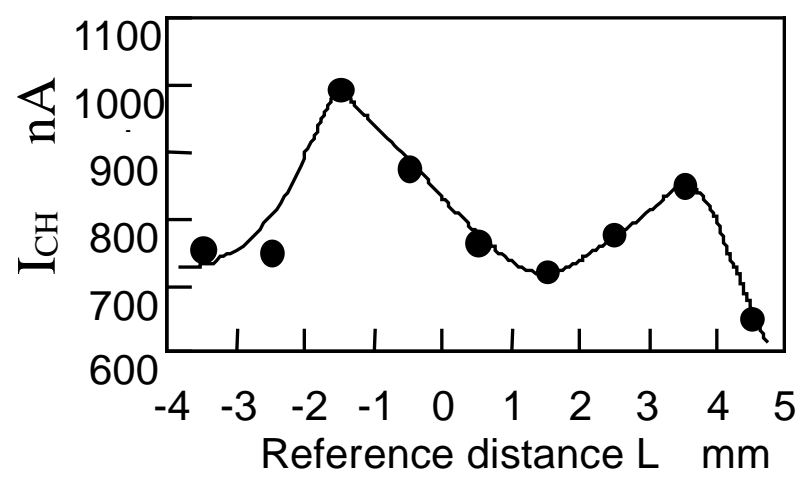

(c) $\mathrm{CH}$-band emissions

Figure 7 Mean signal intensities of Mie scattering, $\mathrm{OH}-$ and $\mathrm{CH}-$ band emission.

part of premixed spray stream is caused by the rapid flame propagation, it is essential to observe the movement of spray boundary. If we apply the PIV method to spray flames, particularly to the vicinity of spray boundary, we must consider the meaning of the results obtained. When the reference circles of cross correlation include the spray boundary, the most influencing factor on the calculated vector is the movement of spray boundary, that is nothing to do with the flow 


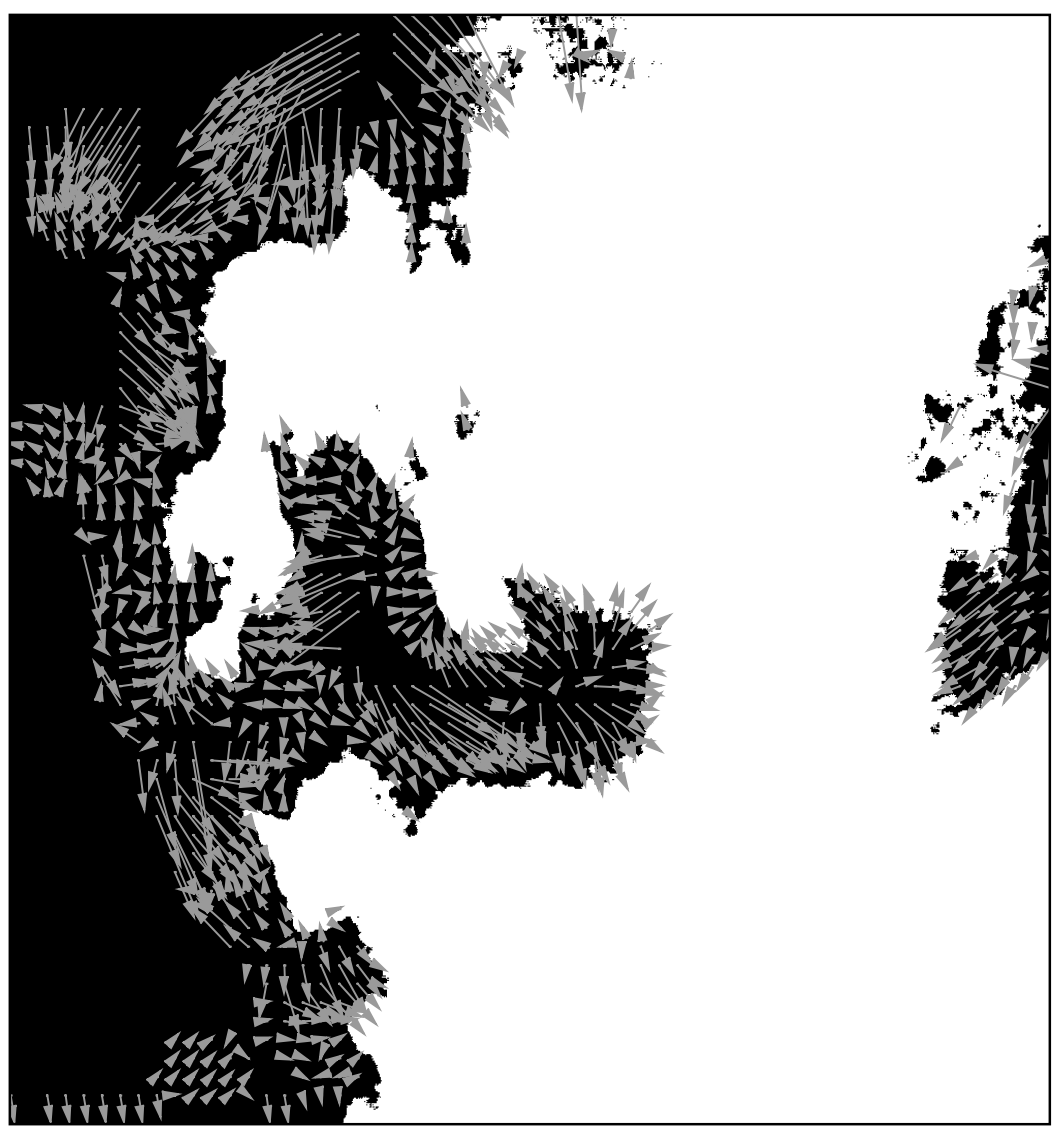

Figure 8 Instantaneous relative velocity vectors against the mean velocity of spray region.

velocity, although some influence of fluid flow might be included as well.

According to Figure 7(a), there are some droplets flowing outside of the binarized spray boundary. Thus, we carefully applied the PIV method again only to those droplets to discuss the motion of spray boundary and fluid motion in the outer vicinity of spray boundaries. In data processing, the time-averaged value of all velocity vectors inside the spray region over 300 images was subtracted from each individual vector outside the spray boundary. Therefore, the obtained vectors in Figure 8 exhibit the relative velocity to the boundary of spray region. Many of vectors in the vicinity of the spray boundary have the component perpendicular to the boundary, probably corresponding to the propagating flame velocity accelerated, to some extent, by the expansion of fluid due to combustion. The profile of mean normal relative velocity, $\mathrm{V}_{\text {norm}}$, in terms of the reference distance from spray boundary is shown in Figure 9 . The positive sign of $\mathrm{V}_{\text {norm }}$ denotes the orientation against the boundary. The figure shows that $\mathrm{V}_{\text {norm }}$ reaches its maximum at around $1.5 \mathrm{~mm}$ away from the spray boundary, where the highest chemiluminescence was observed in Figure 7. It seems an important fact that the movement of spray boundary is related to the local combustion reaction. Therefore, the observed apparent rapid propagation of flame is considered to be the flame propagation accelerated by the local volumetric expansion of fluid due to heat release. 


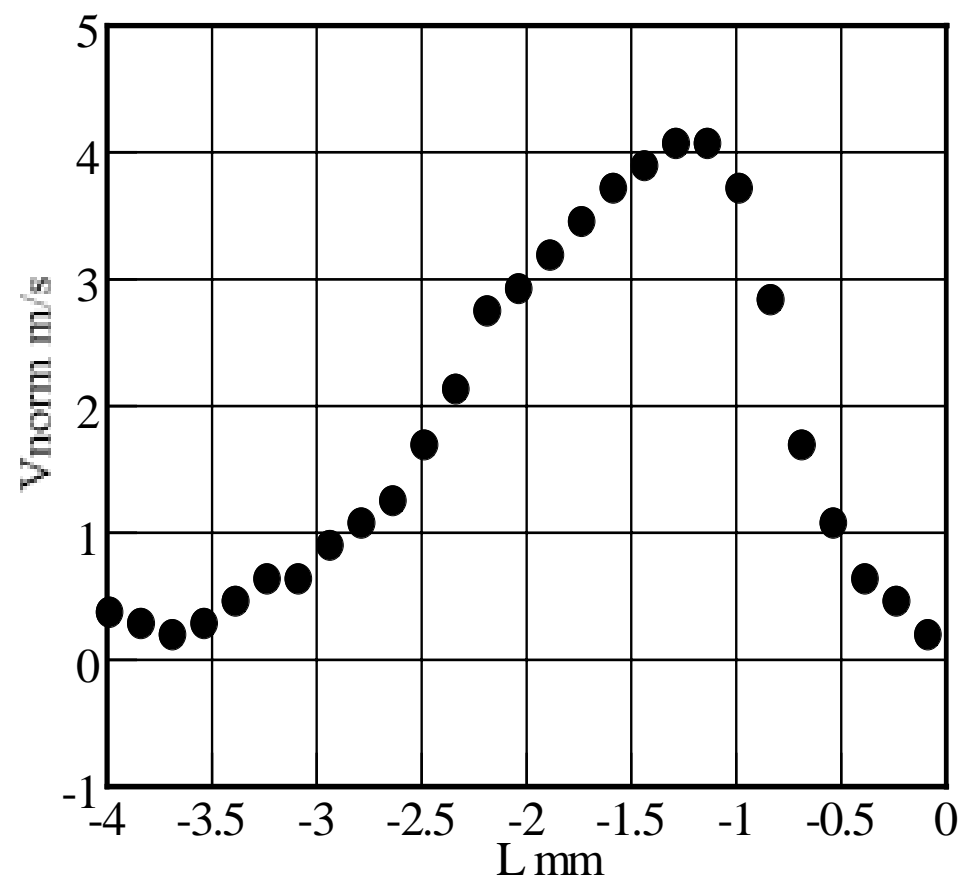

Figure 9 Mean normal relative velocity to spray boundary in terms of the reference distance from the boundary.

\section{CONCLUSIONS}

In order to clarify the influence of turbulent motion on rapid disappearance of the premixedspray stream, particle image velocimetry (PIV) consisting of an Argon-ion laser and a digital CCD camera coupling with an image intensifier were applied to the premixed-spray burner. As a result, no turbulent motion associated with large eddies were identified when a part of premixed-spray stream was abruptly eroded. It was thus confirmed that flames preferentially propagated into an easy-to-burn region of the premixed-spray stream which was named preferential flame propagation in the previous study.

In order to see the structure of propagating spray flame in the direction of propagation, OH- and $\mathrm{CH}-$ band emissions and Mie scattering were measured by MICRO probe together with spray images. It was confirmed that the binarized image of spray boundary coincided with the main vapourization plane of droplets in the flame, and that two distinct reaction regions existed on both sides of the main vaporization region. The frequent and rapid disappearance of a part of premixed-spray stream is considered to be the flame propagation accelerated by local volumetric expansion of fluid due to preferential flame propagation.

\section{ACKNOWLEDGEMENTS}

This research was partially supported by the Grant-in-Aid for Scientific Research, the Ministry 
of Education, Science and Culture, Japan. One of the authors (Tsushima) would like to acknowledge the financial support of Japan Society for the Promotion of Science through Research Fellowships for Young Scientists.

\section{REFERENCE}

Akamatsu, F., Mizutani, Y., Katsuki, M., Tsushima, S. Choi, Y. D. and Nakabe, K., Atom. Sprays, 7-2:199-218(1997).

Akamatsu, F., Wakabayashi, T., Tsushima, S., Katsuki, M., Mizutani, Y., Ikeda, Y., Kawahara, N. and Nakajima, T., Meas. Sci. Tech., 10:1240-1246(1999).

Chew, T. C. Britter, R. E. and Bray, K. N. C., Combust. Flame, 75:165(1989).

Continillo, G. and Sirignano, W. A., Combust. Flame, 81:325(1990)

Gaydon, A. G., The Spectroscopy of Flames, Chapman and Hall, London, 1974.

Greenberg, J. B., Silverman, I. and Tambour, Y., Combust. Flame, 104:358-368(1996).

Hayashi, S., Kumagai, S. and Sakai, T., Combust. Sci. Tech, 15:169-177(1976).

Li, S. C. and Williams, F. A., Proc. Combustion Inst., Vol.26, (1996), p.1017.

Myers, G. D. and Lefebvre, A. H., Combust. Flame, 66:193-210(1986).

Richards, G. A. and Lefebvre, A. H., Combust. Flame, 78:299-397(1989).

Roth, N., Karl, A., Anders, K. and Frohn, A., Proc. Combustion Inst., Vol.26, (1996), p.1697.

Tsushima, S., Saitoh, H., Akamatsu, F. and Katsuki, M., Proc. Combustion Inst., Vol.27, (1998), p.1967. 